\title{
A SINGULAR PERTURBATION METHOD. PART I*
}

\author{
BY \\ N. D. FOWKES \\ Harvard University
}

\begin{abstract}
An approach to singular perturbation problems, introduced by Mahony [1] which arose out of the consideration of a problem involving a boundary layer is applicable to other singular perturbation problems. It lends itself particularly well to problems involving wave propagation, where "multiple scales" are involved. In this paper and the paper to follow, interest is centered around the equation

$$
\epsilon^{3} \nabla^{2} \psi-g(\mathbf{x}) \psi=0,
$$

where $\epsilon$ is a small positive parameter and $g(\mathbf{x})$ is a bounded function of $\mathbf{x}$ which vanishes along simple closed curves in the solution domain. The one-dimensional case (the Langer turning point problem) is considered in this paper and it will be shown that the approach leads to exactly the same results as obtained by Langer and his associates using a "related equation" method.

As far as the author is aware, this is the only nontrivial case in which it can be displayed rigorously (a la Langer and others) that a multiple scaling approach leads to the correct result. The approach employed here, unlike the "related equation" technique, is available in nonlinear and partial differential equation applications, and with this work as background one may be confidently hopeful that the approach will also lead to the correct results in these more complicated problems, and in particular in the partial differential equation application of Part II. In the remaining section of this paper techniques which will be used in Part II are developed in the simpler context, again in the hope that confidence will be gained in the validity of the results obtained using these techniques.
\end{abstract}

1. Introduction. If the solution of a differential equation with parametric dependence on a small number differs significantly from the unperturbed solution (i.e., the solution obtained by equating the parameter to zero), then the problem is referred to as a singular perturbation problem. In the particular case in which the small parameter appears as a coefficient of one of the highly differentiated terms of the equation, the solution may exhibit oscillatory $(e g . \sin x / \epsilon$ ) or exponential (boundary layer) type dependence (eg. exp $-|x| / \epsilon)$ on the small parameter, and entirely different solution procedures have been developed to cope with these two cases. In both these cases a straightforward perturbation approach ${ }^{1}$ fails to yield an interpretable result because the small parameter plays the role of a second solution scale in the problem-not just a solution perturbation parameter. The boundary layer case is essentially the degenerate case in which the second scale is felt only near the boundaries. It will be seen that one approach recently developed by Mahony enables one to deal with both these cases.

*Received February 22, 1967. This research was supported in part by the Office of Naval Research under Contract Nonr-1866(20), and by the Division of Engineering and Applied Physics, Harvard University.

${ }^{1} y(x, \epsilon)=y_{0}(x)+\epsilon y_{1}(x)+\cdots$ 
The one-dimensional wave propagation problems represented by the equation

$$
\epsilon^{3} y^{\prime \prime}-g(x, \epsilon) y=0,
$$

where $\epsilon$ is a small positive parameter, form a class of oscillatory type problems for which suitable techniques have been developed. The methods employed, however, have leant strongly on the linearity and one-dimensionality of this equation-requirements which are not necessary for the application of the method to be employed here. Sturm-Liouville theory indicates that the solutions are highly oscillatory in a domain in which $g(x, \epsilon)$ is negative, and exponential in a domain in which $g(x, \epsilon)$ is positive. In the W.K.B.J. approach solutions of the form $\exp \left(f(x, \epsilon) / \epsilon^{3 / 2}\right)$ are sought, and a direct substitution of this expression into equation (1.1) leads to the W.K.B.J. approximations

$$
y \sim g^{-1 / 4} \exp \epsilon^{-3 / 2}\left( \pm \int g^{1 / 2} d x\right) .
$$

Solution difficulties might be expected in the neighborhood of a zero of $g(x, \epsilon)$, which has been referred to as a turning point, because of the radical change in the solution form across this point. Evidently the solution approximations (1.2) cease to be valid in the neighborhood of such a point, because of the singularity caused by the $g^{-1 / 4}$ factorEq. (1.1) is regular at a zero of $g(x, \epsilon)$, so the exact solution must be finite there. More generally, any point at which the W.K.B.J. approximations cease to be valid solution representations has been referred to as a transition point. Methods have been devised for obtaining connecting relations between the W.K.B.J. approximations valid on either side of a transition point. Modern work on Eq. (1.1), however, has been based on a series of papers by Langer [2], in which he expounded his "comparison" or "related" equation technique. Langer's approach is rather different from that of earlier authors. Instead of trying to connect together the W.K.B.J. approximations across transition points, he sought a single asymptotic expansion valid throughout the solution domain. Transformations of both dependent and independent variables are sought to convert the given equation into one differing only a little from a convenient "comparison" equation whose solutions are known. If the "difference" between these two equations is sufficiently small, a solution of this transformed equation can be shown to be asymptotically approximated by a solution of the comparison equation.

In the field of hydrodynamics, nonlinear partial differential equations are encountered, and the solutions exhibit boundary layer dependence on the small parameter. Prandtl recognized the need for a stretched co-ordinate solution description in the boundary layer. Kaplun and Lagerstrom [3], Proudman and Pearson [4], first devised a suitable systematic method for connecting the boundary layer (or inner) expansion to the expansion valid throughout the remainder of the field (the outer expansion). This they did by rearranging and comparing the terms of the boundary layer and outer expansionsa limiting procedure being employed to determine the relative orders of magnitude of these terms. By removing from the boundary layer expansion the terms in common with the outer expansion, and adding the resulting expression to this outer expansion, a "composite" expansion describing the solution behaviour throughout the solution domain may be obtained. The fact that the matching technique employs a limiting process to compare the relative magnitudes of terms, restricts the parameter range for which the approximations obtained are useful. For example, a term of order $\exp (-h / \epsilon)$ is judged small in comparison with a term of order $\epsilon$, when the limit as $\epsilon \rightarrow 0$ is used as a basis 
for comparison. Numerically, this may not be so for values of $\epsilon$ for which it is desired to apply the solution expansion-especially if $h$ is rather small. Since the limiting procedure must be discarded to overcome this difficulty, the concept of the two expansion regions must also be discarded, and a single expansion valid throughout the complete solution domain must be sought. The terms of this expansion will also be required to decrease for small $\epsilon-$ not only decrease in the limit as $\epsilon \rightarrow 0$ as before. With this in mind Mahony [1] introduced a general singular perturbation approach which enables one to obtain an apparently uniformly valid expansion and applied this approach to a problem arising from the theory of large deflections of an elastic plate. Firstly, the composite expansion is obtained. An attempt is then made to extend the domain of validity of this local expansion. This is done by heuristically "summing" terms of the expansion which would otherwise grow and thus give rise to trouble if the range of interest were extended. Considerations of this kind suggest a suitable expansion modification. The expansion form thus suggested is formally substituted into the equations and, if necessary, the "summation" process is repeated and further modifications made in this way until the complete solution domain is covered. In appropriate cases the summation process indicates the necessity for the inclusion of further scales in the solution description; in other cases more subtle changes are indicated. Cochran [13], and Cole and Kevorkian [14] independently (in a different context) advanced the "multiple scale" idea and arrived at essentially the same "multiple scale" method as that thrown up by Mahony as a biproduct of this approach. It will be shown here that this approach leads to useful solution expansions in oscillatory type problems.

Both the Langer and the Mahony approaches lead to uniformly valid asymptotic expansions and therefore depend either directly or indirectly on the finding of a suitable dominating differential equation. In the Langer approach, the dominating equation is guessed-a sometimes difficult task even in ordinary differential equations, a virtually impossible task in other cases. In Langer's works [15] "The formalism must be pursued inventively, whereas the lines of the concluding rigorous analysis have been pretty well laid down." The approach used in this paper enables one to deduce the dominating equation. Furthermore, this approach is available in nonlinear and partial differential equation applications. In the simple turning point problem it will be shown, in Sec. 2 of this paper, that this approach leads to the same dominating equation and subsequently to the same result, as obtained by Langer's method. In the remaining section (Sec. 3) the multiple transition point problem is considered briefly and an approach which is needed in the extension work of Part II is discussed. Also included in this final section is a brief discussion of a problem which arose out of a stability question, and which is also interesting here because it is related to the "helium molecule ion problem". The main reason for including a brief discussion of this problem here is to indicate the ease and quickness with which one can determine the dominating equation using the above approach.

2. The single, first order turning point problem. Suppose $g(x)$ vanishes at one point, $\alpha$, a simple zero, in the solution domain. Also for the present $g(x)$ will be assumed analytic in the solution domain which will be assumed finite. Both these conditions may be relaxed considerably in the final result.

The preliminary discussion of the introduction indicated that the transition point

2Often referred to as the "multiple timing" method. 
is a special point in the solution domain. It seems natural, therefore, to commence by looking for solutions in the neighborhood of the transition point $\alpha$. Now $g(x)$ is analytic and vanishes at $\alpha$, and therefore can be expanded in the form

$$
g(x)=\sum_{1}^{\infty} g_{k}(x-\alpha)^{k},
$$

about $x=\alpha$. For convenience it is assumed here that $g_{1}=1$, a condition which can be arranged by a suitable choice of scales.

A balance is achieved between the two terms of Eq. (1.1) in the neighborhood of $x=\alpha$ if significant variations of $y$ occur in a range of $x$ of order $\epsilon$. This suggests the introduction of the stretched co-ordinate $\eta=(x-\alpha) / \epsilon$, in terms of which the expansion (2.1) becomes

$$
g(x)=\sum_{1}^{\infty} \epsilon^{k} \eta^{k} g_{k}
$$

Equation (1.1), when expressed in terms of $\eta$ becomes

$$
y_{\eta \eta}-\eta y=y \sum_{2}^{\infty} \epsilon^{k-1} g_{k} \eta^{k} .
$$

The expansion form,

$$
y=y_{0}(\eta)+\epsilon y_{1}(\eta)+\cdots,
$$

suggested by Eq. (2.2) certainly cannot be expected to be useful for nonsmall values of $(x-\alpha)$ because the expansion (2.1) for $g(x)$ is useless for such values, however, such a solution expansion will have a small range of validity and, therefore, will be an appropriate one to commence an investigation. An examination of this expansion will suggest a modification which will enable an extended domain to be covered.

The first few expansion terms of the "exponentially small" solution are given by,

$$
y=\left[A_{0} A i(\eta)\right]+\epsilon\left[\left(g_{2} A_{0} / 5\right)\left(\eta^{2} A i^{\prime}(\eta)-\eta A i(\eta)\right)+A_{1} A i(\eta)\right]+\epsilon^{2}[\cdots]+\cdots
$$

where $\operatorname{Ai}(\eta)$ is the Airy Bessel function which is exponentially small for large positive $\eta$. Higher order terms in the expansion contain still higher powers of $\eta^{3}{ }^{3}$ It is clear from (2.4) that the expansion (2.3) ceases to be useful for values of $\eta$ greater in order than $\left(1 / \epsilon^{1 / 2}\right)$ (corresponding to a range of $(x-\alpha)$ of order $\left.\epsilon^{1 / 2}\right)$. In order to obtain a first term $y_{0}{ }^{*}$ which would dominate the solution behaviour for values of $(x-\alpha)$ of unit order it would be necessary to add to the $y_{0}$ above, terms occurring later in the present expansion for $y$ which, although unimportant for small values of $(x-\alpha)$, assume importance for values of $(x-\alpha)$ of unit order. To obtain some idea of the type of adjustment required the sum of the first two terms of the present expansion (2.4) is considered.

Now, because overestimation of the order of a term is not serious whereas underestimation is, $\eta^{2} A i^{\prime}(\eta)$ is better represented in the form $\epsilon^{-2}(x-\alpha)^{2} A i^{\prime}(\eta)$ over a range of $(x-\alpha)$ of unit order. Thus for values of $(x-\alpha)$ of unit order, the sum (2.4) is better represented in the rearranged form

$$
y_{0}+\epsilon y_{1}=A_{0} A i(\eta)+\frac{(x-\alpha)^{2}}{\epsilon} \frac{g_{2} A_{0}}{5} A i^{\prime}(\eta)-\frac{g_{2} A_{0}}{5}(x-\alpha) A i(\eta)+\epsilon A_{1} A i(\eta) .
$$

${ }^{3} y_{n}$ contains terms like $\eta^{2 n}\left(A i^{\prime}(\eta)\right)+\cdots \eta^{2 n-1}(\cdots)$ etc. 
This expression can be written in the "summed" form

$$
\begin{aligned}
y_{0}+\epsilon y_{1}=\left\{A_{0}-\frac{g_{2} A_{0}}{5}\right. & \left.(x-\alpha)+O(x-\alpha)^{2}\right\} \\
\cdot & \left\{A i\left[\frac{(x-\alpha)}{\epsilon}+g_{2} \frac{(x-\alpha)^{2}}{5 \epsilon}+O \frac{(x-\alpha)^{3}}{\epsilon}\right]\right\}+O(\epsilon),
\end{aligned}
$$

i.e.

$$
y_{0}+\epsilon y_{1}=A(x) A i(u(x) / \epsilon)+O(\epsilon)
$$

where

$$
u(x)=(x-\alpha)+g_{2}\left[(x-\alpha)^{2} / 5\right]+\cdots
$$

and

$$
A(x)=A_{0}-\left(g_{2} A_{0} / 5\right)(x-\alpha)+\cdots,
$$

as can be seen by expanding the right hand side of the above expression. This suggests that an extension of the solution domain so far covered by the expansion form (2.3) may be achieved if a solution of the form $y=y(\zeta, x, \epsilon)$, where $\zeta=u(x) / \epsilon,(u(x)$ being suitable chosen) is sought. It should be noted that close to $\alpha, \zeta \sim(x-\alpha) / \epsilon$, and therefore $\zeta$ is expected to differ inessentially from the stretched co-ordinate, $\eta$ there.

Note. The above argument is purely heuristic but its great advantage lies in the fact that it offers a method for seeking out solution forms which may lead to more useful perturbation expansions. The "sum" (2.6) is not the only one suggested by (2.5); however, since many equivalent useful descriptions of the solution behaviour (corresponding to different "sums") are available, and we only seek one of these here, this need not be of concern.

2.1. The Formal Solution. In the hope that the above argument has suggested a useful solution modification, solutions of the form,

$$
y=y(x, \zeta, \epsilon),
$$

where

$$
\zeta=u(x) / \epsilon
$$

a function to be determined in the solution process, are sought. The function $u(x)$ will be referred to in the work to follow as the "boundary layer" function, to correspond with the terminology used by Mahony.

Now, in terms of the assumed form

$$
y^{\prime}(x, \epsilon)=\epsilon^{-1} y_{\zeta}(x, \zeta, \epsilon) u_{x}+y_{x}(x, \zeta, \epsilon)
$$

and

$$
y^{\prime \prime}(x, \epsilon)=\epsilon^{-2} y_{\zeta \zeta}(x, \zeta, \epsilon) u_{x}^{2}+\epsilon^{-1}\left(2 y_{x \zeta}(x, \zeta, \epsilon) u_{x}+y_{\zeta}(x, \zeta, \epsilon) u_{x x}\right)+y_{x x}(x, \zeta, \epsilon)
$$

where ' denotes total differentiation with respect to $x$, and the derivatives on the right hand side are partial derivatives. On substituting (2.9) in Eq. (1.1) the following equation for $y(x, \zeta, \epsilon)$ is obtained;

$$
y_{\zeta \zeta}-\frac{1}{\epsilon}\left(\frac{g}{u_{x}^{2}}\right) y=-\left\{\epsilon\left(2 y_{x \zeta} / u_{x}+y_{\zeta} u_{x x} / u_{x}^{2}\right)+\epsilon^{2} y_{x x} / u_{x}^{2}\right\} .
$$


The "boundary layer" function was introduced to account for the solution behavior in $\epsilon$ neighborhood of $x=\alpha$. In this neighborhood $g(x)$ is of order $\epsilon$, and therefore $\left(\epsilon^{-1} g(x) / u_{x}^{2}\right)$ is of unit order. An equivalent (see (2.8)) though better representation (in terms of magnitude) of this term is therefore given by $\left((\zeta / u) g(x) / u_{x}^{2}\right)$. Equation $(2.10)$ now becomes

$$
y_{\zeta \zeta}-k^{3}(x) \zeta y=-\left\{\epsilon\left(2 y_{x \zeta} / u_{x}+y_{\zeta} u_{x x} / u_{x}^{2}\right)+\epsilon^{2} y_{x x} / u_{x}^{2}\right\}
$$

where

$$
k^{3}(x)=g /\left(u u_{x}^{2}\right) .
$$

An asymptotic expansion for $y(x, \zeta, \epsilon)$ of the form

$$
y=\sum_{0}^{\infty} \epsilon^{n} y_{n}(x, \zeta)
$$

suggests itself. On substituting this expression for $y$ in Eq. (2.11), and equating to zero the coefficients of the various powers of $\epsilon$, the following equations are obtained;

$$
\begin{aligned}
& y_{055}-k^{3} \zeta y_{0}=0 \\
& \left.y_{n \zeta 5}-k^{3} \zeta y_{n}=-\left\{\left(2 y_{n-1 x \zeta} / u_{x}+y_{n-1 \zeta} u_{x x} / u_{x}^{2}\right)+y_{n-2 x x} / u_{x}^{2}\right\} \quad \text { (for } n \geq 1\right)
\end{aligned}
$$

where $y_{n}$ is interpreted to be zero for negative $n$.

Equation (2.14a) has as independent solutions, $A_{0}(x) A i(k \zeta)$ and $B_{0}(x) B i(k \zeta)$, where $A_{0}(x)$ and $B_{0}(x)$ are, as yet, undetermined functions of $x$. Because the equations (2.14b) are linear in $y_{n}, y_{n-1}$, and $y_{n-2}$, these independent leading terms in the expansion for $y$ give rise to independent higher order terms in the expansion for $y$. The solutions, exponentially small for large positive $\zeta$, corresponding to a first expansion term of the form

$$
y_{0}=A_{0}(x) A i(k \zeta)
$$

will be sought. The other independent solutions based on $B i(k \zeta)$ can be found in the same way. On substituting (2.15) into Eq. (2.14b) with $n=1$, the equation for $y_{1}$ becomes

$$
y_{1 \zeta \zeta}-k^{3} \zeta y_{1}=-\left\{F\left(A_{0} k\right) A i^{\prime}(k \zeta)+k_{x}\left(A_{0} k^{4}\right) \zeta^{2} A i(k \zeta)\right\},
$$

where $F(\gamma)$ denotes

$$
\frac{2}{u_{x}} \frac{d}{d x}(\gamma)+\frac{u_{x x}}{u_{x}^{2}} \gamma
$$

Equation (2.16) is effectively an ordinary differential equation in $\zeta$, with $x$ as an independent parameter. Its solution is the sum of a complementary function and an independent particular integral. This particular integral contains terms of the form $\zeta^{2} A i^{\prime}(k \zeta)$ and $A i(k \zeta)$, arising out of the $\zeta^{2} A i(k \zeta)$ portion of this equation, and a term of the form $\zeta A i(k \zeta)$ arising out of the $A i^{\prime}(k \zeta)$ portion of this equation. Thus, in the solution for $y_{1}$, terms in $\zeta^{2} A i^{\prime}(k \zeta)$ and $\zeta A i(k \zeta)$ arise, which correspond to the objectionable ${ }^{4}$ terms, $\eta^{2} A i^{\prime}(\eta)$ and $\eta A i(\eta)$, which arose in the heuristic development of Sec. (2.1) (see Eq. (2.4)). In the present development, a considerable amount of freedom is present

'Objectionable because the expansion, $y=y_{0}+\epsilon y_{1}$ etc., is not asymptotic for nonsmall values of $(x-\alpha)$ because of the presence of these terms. 
in the solution form, for neither $u(x)$ nor $A_{0}(x)$ are yet determined. This freedom is now utilized to remove these objectionable terms.

By equating $k_{x}$ to zero, the $\zeta^{2} A i(k \zeta)$ term of Eq. (2.16) is removed and the boundary layer function $u(x)$ is defined. For if $k_{x}=0$ i.e. $k=$ constant, 1 (say); ${ }^{5}$ then from (2.12),

$$
u u_{x}^{2}=g \text {. }
$$

Now, if $u$ is to behave like $g$ close to $x=\alpha$, (and this is suggested by the heuristic development of the previous section) then $u$ must be the real solution of this equation. Clearly, for real solutions of Eq. (2.18), $u(x)$ and $g(x)$ have the same sign throughout the solution domain, and therefore $u(x)$ vanishes at $x=\alpha$, the zero of $g(x)$. This, together with Eq. (2.18) and the requirement that $u(x)$ have a continuous first derivative ${ }^{6}$ determines the boundary layer function $u(x)$, as

$$
u(x)=\operatorname{sign}(g(x))\left\{\frac{3}{2}\left|\int_{\alpha}^{x}(|g(x)|)^{1 / 2} d x\right|\right\}^{2 / 3} .
$$

Now that the $\zeta^{2} A i(k \zeta)$ term has been removed from Eq. (2.16) the only objectionable term remaining is the $A i^{\prime}(k \zeta)$ term and this can be removed by simply requiring that its coefficient $F\left(A_{0}\right)$ vanish identically. ${ }^{7}$ Thus,

$$
\left.O=F\left(A_{0}\right)=\frac{2}{u_{x}} \frac{d A_{0}}{d x}+\frac{u_{x x}}{u_{x}^{2}} A_{0} \quad \text { (from } \quad(2.17)\right) .
$$

This is a first order, linear, differential equation from which $A_{0}(x)$ can be determined as

$$
A_{0}(x)=\frac{K_{0}}{\left|u_{x}\right|^{1 / 2}},
$$

where $K_{0}$ is an arbitrary constant of integration. As far as the particular solution of Eq. (1.1) is concerned, $K_{0}$ is nothing more than the usual arbitrary multiplicative constant associated with the solution of a linear homogeneous differential equation. The evaluation of $K_{0}$ is therefore to be achieved when suitable complete boundary conditions are imposed on the solution, $y$. Now since $g(\alpha)$ has a simple zero at $x=\alpha, u_{x}(\alpha)$ (from $(2.19)$ ) is bounded from zero. Also, $u(x)$ is strictly monotonic as is seen from its integral representation (2.19) so that (from Eq. (2.18)) $u_{x}(x)$ is bounded from zero. Thus, $A_{0}(x)$ and its derivatives are certainly bounded for the analytic functions, $g(x)$, considered in this development.

Now that $A_{0}(x)$ and $u(x)$ have been determined in the manner described above, all terms on the right hand side of Eq. (2.16) vanish identically so that this equation now integrates to give

$$
y_{1}=A_{1}(x) A i(\zeta)
$$

where $A_{1}(x)$ is, as yet, an arbitrary function of $x$. This expression for $y_{1}$ fits into the asymptotic scheme for values of $x$ of unit order and indeed for all values of $x$ in the solution domain ${ }^{8}$ provided that $A_{1}(x)$, when determined, is bounded.

'There is no loss in generality in choosing this constant unity.

It is convenient, though not necessary, to remain on the one solution branch of (2.18) in this way. 'The reader will recall that $A_{0}(x)$ is as yet undetermined.

${ }^{8}$ Which may be infinite. 
The equation for $y_{2}$ now reduces to

$$
y_{2 \zeta \zeta}-\zeta y_{2}=-\left\{A i^{\prime}(\zeta) F\left(A_{1}\right)+A i(\zeta) A_{0}^{\prime \prime}(x) / u_{x}^{2}\right\}
$$

The particular integral associated with $A i(\zeta)$ is $A i^{\prime}(\zeta)$. Thus the particular integral associated with $A i(\zeta)$ term of Eq. (2.22) fits into the asymptotic scheme. Again the $A i^{\prime}(\zeta)$ term is required to vanish identically which requires $F\left(A_{1}\right)=0$. This determines $A_{1}(x)$ as

$$
A_{1}(x)=K_{1} /\left|u_{x}\right|^{1 / 2},
$$

which is bounded as required. The constant of integration $K_{1}$, again is determined when suitable boundary conditions are imposed on the solution, $y$. With $A_{1}(x)$ determined by (2.23), the equation for $y_{2}$ can be integrated to give

$$
y_{2}=A_{2}(x) A i(\zeta)-A i^{\prime}(\zeta)\left(A_{0}^{\prime \prime}(x) / u_{x}^{2}\right)
$$

The equation for $y_{3}$ now becomes

$$
y_{3 \zeta \zeta}-\zeta y_{3}=-A i^{\prime}(\zeta) F\left(A_{2}\right)-A i(\zeta)\left(A_{1}^{\prime} / u_{x}^{2}\right)+A i^{\prime \prime}(\zeta) F\left(A_{0}^{\prime \prime} / u_{x}^{2}\right) .
$$

The particular integral associated with $A i^{\prime \prime}(\zeta)$ is $(2-\zeta) A i^{\prime}(\zeta)$, which, for values of $x$ of unit order, is better represented in magnitude in the form $\left(2 A i^{\prime}(\zeta)-\epsilon^{-1} u(x) A i^{\prime}(\zeta)\right)$. The particular integral arising out of the $A i^{\prime \prime}(\zeta)$ term of Eq. (2.25), therefore, does not fit into the asymptotic scheme. Thus, both the $A i^{\prime}(\zeta)$ term and the $A i^{\prime \prime}(\zeta)$ term give rise to unsuitable particular integrals. The particular integral terms corresponding to these cannot cancel one another in other than a very restricted region because their functional forms are different. Thus the two terms must be coped with independently. As before the $A i^{\prime}(\zeta)$ term can be removed by requiring that its coefficient vanish identically which determines $A_{2}(x)$ as

$$
A_{2}(x)=K_{2} /\left|u_{x}\right|^{1 / 2}
$$

The $A i^{\prime \prime}(\zeta)$ term in Eq. (2.25) cannot be dealt with so simply however. The coefficient of $A i^{\prime \prime}(\zeta)$ in this equation is completely determined, and in general does not vanish identically. Now,

$$
A i^{\prime \prime}(\zeta)=\zeta A i(\zeta)^{9}=(u(x) / \epsilon) A i(\zeta),
$$

and herein the trouble lies; for it is now clear that the $A i^{\prime \prime}(\zeta)$ term on the right-hand side of Eq. (2.25) for $y_{3}$ is of order $\epsilon^{-1}$ and, therefore, should have appeared one stage earlier in the solution proceedings (i.e. in the Eq. (2.22) for $y_{2}$ ). It is thus apparent that the formal solution process, as described by $(2.14 \mathrm{~b})$, has led to an unsatisfactory arrangement of terms and that an alternative arrangement must be sought. Now, since the difficult term $\left(A i^{\prime \prime}(\zeta) F\left(A_{0}^{\prime \prime} / u_{x}^{2}\right)\right)$ in Eq. (2.25) for $y_{3}$ can be rewritten in the equivalent form $\left(\epsilon^{-1} u(x) A i(\zeta) F\left(A_{0}^{\prime \prime} / u_{x}^{2}\right)\right)$ this suggests the rearrangement based on simply including this term as an additional term in the Eq. (2.22) for $y_{2}$-thereby modifying the schematic solution process as described by Eq. (2.14b). Now the particular integral associated with $A i(\zeta)$ is $A i^{\prime}(\zeta)$ so that the extra term included in the equation for $y_{2}$ will give rise to an extra $A i^{\prime}(\zeta)$ term $\left(\left[F\left(A_{0}^{\prime \prime} / u_{x}^{2}\right) u(x)\right] A i^{\prime}(\zeta)\right)$ in the solution for $y_{2}$. This, in turn, will give rise to an extra $A i^{\prime \prime}(\zeta)$ term $\left(f_{1}(x) A i^{\prime \prime}(\zeta)\right.$ (say)) in the Eq. (2.25) for $y_{3}$, which, as before, is rewritten in its equivalent $\left(\epsilon^{-1} u(x) f_{1}(x) A i(\zeta)\right)$ form and is included in the

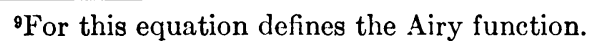


equation for $y_{2}$ to give rise to still another $A i^{\prime}(\zeta)$ term $\left(\left[f_{1}(x) u(x)\right] A i^{\prime}(\zeta)\right)$ in the solution for $y_{2}$, and so on. Thus extra $A i^{\prime}(\zeta)$ terms in $y_{2}$ are generated successively and an expression for $y_{2}(x, \zeta)$ of the form,

$y_{2}(x, \zeta)=A_{2}(x) A i(\zeta)+A i^{\prime}(\zeta)\left(F\left(A_{0}^{\prime \prime} / u_{x}^{2}\right) u(x)+f_{1}(x) u(x)+f_{2}(x) u(x)+\cdots\right)$

results. The process of simply transferring large terms (the $A i^{\prime \prime}(\zeta)=\epsilon^{-1} u(x) A i(\zeta)$ terms) in the equation for $y_{3}$ back into the equation for $y_{2}$ as described above, therefore, does not lead to a useful result (the successive coefficients of $A i^{\prime}(\zeta)$ in (2.26a) do not decrease). The form of this expression (2.26a) for $y_{2}$, however, suggests that the appropriate "sum" is of the form,

$$
y_{2}(x, \zeta)=A_{2}(x) A i(\zeta)+\alpha_{2}(x) A i^{\prime}(\zeta),
$$

where clearly $\alpha_{2}(x)$ must be determined in such a way that the successive generation of extra terms in the equation for $y_{2}$ is avoided. With $y_{2}$ so defined (where $\alpha_{2}(x)$ as yet undetermined) the equation for $y_{3}$ becomes,

$$
y_{3 \zeta \zeta}-\zeta y_{3}=-A i(\zeta) A_{1}^{\prime \prime}(x) / u_{x}^{2}-A i^{\prime \prime}(\zeta) F\left(\alpha_{2}\right),
$$

(where $F\left(A_{2}\right)$ has been required to vanish as before) and the term now to be transferred back into the equation for $y_{2}$ is $-A i^{\prime \prime}(\zeta) F\left(\alpha_{2}\right)$. When this term is written in the form $\epsilon^{-1} u(x) A i(\zeta) F\left(\alpha_{2}\right)$ and is included in the equation for $y_{2}$ this equation becomes,

$$
\begin{aligned}
y_{25 \zeta}-\zeta y_{2} & =-A i(\zeta) A_{0}^{\prime \prime} / u_{x}^{2}-A i(\zeta)\left(u(x) F\left(\alpha_{2}\right)\right) \\
& =-A i(\zeta)\left(A_{0}^{\prime \prime} / u_{x}^{2}+u(x) F\left(\alpha_{2}\right)\right)
\end{aligned}
$$

Now $y_{2}$ as given by (2.27) satisfies this equation if $\alpha_{2}(x)$ is defined to satisfy,

$$
u F\left(\alpha_{2}\right)+\alpha_{2}+A_{0}^{\prime \prime} / u_{x}^{2}=0
$$

By defining $\alpha_{2}(x)$ in this way the successive generation is automatically avoided. Thus by simply modifying the schematic process as described by (2.14b) in the way indicated above the $A i^{\prime \prime}(\zeta)$ difficulty is overcome.

Equation (2.30) is a linear first order differential equation in $\alpha_{2}(x)$ whose general solution

$$
\alpha_{2}(x)=\exp \left(-\int p(x) d x\right) \int_{x_{0}}^{x} q(x) \exp \left(\int p(x) d x\right) d x,
$$

where $p(x)=\left(u_{x x} / u_{x}^{2}+1 / u\right) u_{x} / 2$, and

$$
q(x)=-\left(u_{x} / 2\right) A_{0}^{\prime \prime}(x) / g(x),
$$

and $x_{0}$ is arbitrary. An examination of this expression shows that $\alpha_{2}(x)$ has bounded derivatives of all order throughout the solution domain if, and only if, $\alpha_{2}(x)$ vanishes at $x=\alpha$. This property is required to ensure the higher terms on the expansion for $y$ are bounded. Thus $\alpha_{2}(x)$ is uniquely determined as

$$
\alpha_{2}(x)=\exp \left(-\int p(x) d x\right) \int_{\alpha}^{x} q(x) \exp \left(\int p(x) d x\right) d x .
$$

The uniqueness of $\alpha_{2}(x)$ is to be expected because only one arbitrary constant can arise in the expression, (2.27), for $y_{2}$, and this has already arisen in the $A i(\zeta)$ term. 
With $\alpha_{2}(x)$ determined as above, the equation for $y_{3}$ now becomes

$$
y_{3 \zeta \zeta}-\zeta y_{3}=-\left(A_{1}^{\prime \prime}(x) / u_{x}^{2}\right) A i(\zeta),
$$

which, in essence, is the same as the equation for $y_{2}$ as it appeared in (2.22). The process as it has been described above can therefore be repeated formally ad infinitum since no new type of dependence on $\zeta$ will appear on any right hand side. In the application of this method to less tractable equations new types of $\zeta$ dependence will appear at each stage of the solution process so that the problem of determining the higher order solution terms will become successively more complicated, but in the present example since the particular integrals will involve $\zeta$ only through $A i^{\prime}(\zeta)$ it is convenient to take advantage of this simplicity of form and derive the successive terms by formal substitution of the expression

$$
y=A i(\zeta) \sum_{0}^{\infty} \epsilon^{n} A_{n}(x)+A i^{\prime}(\zeta) \sum_{2}^{\infty} \epsilon^{n} \alpha_{n}(x),
$$

in the differential Eq. (2.11) and equating the coefficients of $A i(\zeta)$ and $A i^{\prime}(\zeta)$ to zero. This leads to the following ordinary linear differential equations for $A_{m}$ and $\alpha_{n}$ which can be solved successively;

$$
F\left(A_{n}\right)+\alpha_{n-1}^{\prime}(x) / u_{x}^{2}=0
$$

and

$$
\alpha_{n}(x)+u F\left(\alpha_{n}\right)+A_{n-2}^{\prime \prime}(x) / u_{x}^{2}=0
$$

with $\alpha_{n}(\alpha)=0$ for $n \geq 3$.

The exponentially large solution is simply obtained by replacing $A i(\zeta)$ by $B i(\zeta)$ and $A$ 's and $\alpha$ 's in the above expressions by $B$ 's and $\beta$ 's.

This is exactly the same result as that obtained by Olver [5] who used a "related equation" technique. Olver [5], Langer [2], and others have shown that under fairly general conditions, this is indeed an asymptotic representation of the solution of Eq. (1.1) which is exponentially small for large positive $x$. Thus here is a nontrivial case (i.e. not constant coefficients) where an answer derived by a multiple scaling method has been shown (by an independent indirect investigation) to be a genuine asymptotic representation of the exact solution over a significant range in both scales. As far as the author is aware, this is the only nontrivial case in which one has been able to illustrate that "multiple scaling" does lead to the correct result. Thus, with some justification, one may be confidently hopeful that the results detained in further "multiple scale" applications, and in particular the partial differential equation application of Part II will be correct.

The important thing to note about the approach presented here is that it enables one to deduce the correct "dominating" or "related" equation. In the "related equation" approach the "related" equation is guessed. In Langer's own words [15] "The formalism must be pursued inventively whereas the lines of the concluding rigorous analysis have been pretty well laid down." The fact that

$$
y_{5 \zeta}-\zeta y=0 .
$$

with $\zeta$ defined by Eq. (2.19), is the appropriate dominating or "related equation" follows immediately from Eq. (2.11) since none of the "leftover" terms in this equation

$$
y_{\zeta \zeta}-\zeta y=\epsilon\left(2 y_{x \zeta} / u_{x}+y_{\zeta} u_{x x} / u_{x}^{2}+\epsilon y_{x x} / u_{x}^{2}\right),
$$


are greater in order than $\epsilon$. If one wished, one could at this stage pursue the problem using the Langer integral equation approach [2], however, if one wishes to obtain an asymptotic expansion, then the formal expansion procedure above indicates clearly the stage in the asymptotic expansion at which care must be taken (e.g. the necessity of the $A i^{\prime}(\zeta)$ term is clearly indicated). The approach indicated in this paper also leads to the dominating equation and asymptotic solution expansions in partial differential equations and nonlinear differential equations.

Since the aim here is to illustrate a method rather than establish a result, it suffices here to state that (although rigorous proofs of this result are not yet available) (2.33) appears to be correct for physically interesting functions $g(x)$ in the infinite domain except in very special cases and point out that the Mathieu equation is one such special case. Mahony [12] in a recent paper discusses this point and in particular he considers the Mathieu equation.

3. Further problems. Two problems which are primarily of interest in association with the partial differential equation extension work of Part II are now considered briefly.

3.1. Multiple Transition Point Problems. Uniformly valid asymptotic expansions have been obtained by Langer [7] and Kazarinoff [8] using a "related equation" technique in the two turning points case. Clearly such expansions will be useless if they are not in terms of functions with known properties and which are also well tabulated. The limit of usefulness thus defined is reached at the two transition points cases. ${ }^{10}$ The Airy function expansions of the previous section are valid representations of the solutions of Eq. (1.1) in a domain containing a single turning point so that the possibility of describing the solution behaviour in terms of expansions valid in overlapping domains about each of the turning points is worth investigating. Olver [9] has considered this possibility and has derived connecting relations between the two expansions by tracing particular solutions around in the complex plane. However, this technique is not available in the partial differential equation application of Part II. An alternative simple minded approach which leads to the correct results in this case and which is available in the partial differential equation application will be discussed here. With this work as background, one may be confidently hopeful that the approach will lead to the correct results in the later application. Here the question "Under what conditions will patching (matching the solution and its derivative at a point) lead to a useful result" will be answered. Since different solutions may have exactly the same asymptotic expansion in certain regions in the solution domain, it is reasonable to inquire whether there is anything to be gained by being selective in one's choice of the point of patching.

If the expansions are to be patched in the oscillatory portion of the solution domain (which must be accomplished in the case in which $g<0$ for $\alpha_{1}<x<\alpha_{2}$ ) where both $A i(\zeta)$ and $B i(\zeta)$ are of unit order, then errors which are algebraically small in $\epsilon$ will be picked up in the coefficients of $A i(\zeta)$ and $B i(\zeta)$ due to patching. ${ }^{11}$ The $B i(\zeta)$ error thus introduced will swamp the solution in the exponential solution domain if the solution itself is exponentially small. ${ }^{12}$ In all other cases the patching error can be shown to be relatively unimportant [6]. The potential well problem is a difficult case in point. In this case, since any portion of a $B i(\zeta)$ solution (however small) cannot be allowed, one can

${ }^{10}$ Weber functions arise in the above treatment of the two turning points case.

${ }^{11}$ One can only hope to patch the expansions together up to terms of finite order.

${ }^{12}$ In the exponential region $A i(\zeta)$ is of order $\exp -\left(\frac{2}{3} \zeta^{3 / 2}\right)$ while $B i(\zeta)$ is of order $\exp \left(+\frac{2}{3} \zeta^{3 / 2}\right)$. 
sidestep the above difficulty by ensuring the correct expansion behavior at the two end points $\pm \infty$. The eigencondition requirement,

$$
A i\left(\bar{\zeta}_{1}\right) A i^{\prime}\left(\bar{\zeta}_{2}\right)-A i\left(\bar{\zeta}_{2}\right) A i^{\prime}\left(\bar{\zeta}_{1}\right)=O(\epsilon)
$$

where $\zeta_{1}=\bar{\zeta}_{1} \zeta_{2}=\bar{\zeta}_{2}$ is the patching point, then results from the patching conditions. The independence of this eigencondition on the actual position of the point of patching follows directly from the fact that the left-hand side of the above expression is the Wronskian of a second order ordinary differential equation. Thus, in the potential well

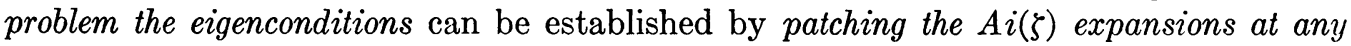
convenient point between the two turning points. ${ }^{13}$ The most convenient point to patch the expansions together is the point given by $\bar{\zeta}=\bar{\zeta}_{1}=\bar{\zeta}_{2}$. The analysis is then simplified because in this case $u_{1}(x)$ and $u_{2}(x)$ are continuous across the patching point. If the expansions are to be patched in the exponential portion of the solution domain (so that $g>0$ for $\alpha_{1}<x<\alpha_{2}$ ) then clearly no hope can be held out if the solution is exponentially large because a very large error will be picked up in the $A i(\zeta)$ (exponentially small) component, and in the oscillatory domain where $A i(\zeta)$ is of unit order this error will swamp the solution itself. If, however, the solution is exponentially small then patching results in a correct answer if and only if the "centre" $\bar{\zeta}_{1}=\bar{\zeta}_{2}=\bar{\zeta}$ (say) is chosen as the patching point. To see this it is necessary to notice first of all that only an exponentially small $B i(\zeta)$ term is allowed and errors introduced in this term are important nowhere, and secondly that exponentially large $A i(\zeta)$ errors will be introduced if patching is undertaken either to the left or to the right of "centre". ${ }^{14}$

3.2. The Dominating Equation. It was pointed out at the end of Sec. 2, that after one has obtained the appropriate expansion form, by either guessing or using the heuristic approach suggested in section 1, one can quickly derive the "related" (or dominating) equation by simply assessing the various terms of the differential equation. To illustrate this the equation

$$
\epsilon^{2} y_{x x}-g(x, \epsilon) y=0,
$$

where $g(x, \epsilon)=f(x)-\epsilon(h(x) / x)$, where $f(x)$ and $g(x)$ are analytic and positive in the domain of interest, is considered. This problem arose out of a stability context but is of interest here because its two dimensional analogue is closely related to a problem that arises when one tries to extract the high energy states of the helium molecule ion. ${ }^{15}$ Now a heuristic discussion similar to that used in section 1 leads to the suggestion that a solution representation of the form $y=y(\zeta, x, \epsilon)$ where $\zeta=u(x) / \epsilon$, an as yet unknown function of $x$, be sought. In terms of $\zeta$ the equation for $y$ becomes,

$$
y_{\zeta \zeta}-\alpha\left(\frac{\alpha}{4}-\frac{\gamma}{\zeta}\right) y=-\epsilon\left(2 y_{x \zeta} / u_{x}+y_{\zeta} \frac{u_{x x}}{u_{x}^{2}}\right)-\epsilon^{2} y_{x x} / u_{x}^{2},
$$

where $\alpha=2 f^{1 / 2} / u_{x}$ and $\gamma=u h /\left(\alpha x u_{x}^{2}\right)$. Now one has to assess the various terms of this equation. The terms that are apparently of order $\epsilon$ do not, in fact, give rise to large

${ }^{13}$ Excluding small neighborhoods of both turning points-the Airy function expansion about a turning point ceases to be valid in the immediate neighborhood of a second turning point.

${ }^{14}$ at $\zeta_{1}=\bar{\zeta}_{1}, A i\left(\zeta_{1}\right)$ is of order $\exp \left(-2 / 3 \bar{\zeta}_{1}^{3 / 2}\right)$ so that an expansion of the form [ $\{A(x, \epsilon) \exp (2 / 3$ $\left.\left.\left.\bar{\zeta}_{2} 2^{3 / 2}-2 / 3 \bar{\zeta}_{1}^{3 / 2}\right) A i\left(\zeta_{2}\right)\right\}+\left\{B(x, \epsilon) \exp -\left(2 / 3 \bar{\zeta}_{2}^{3 / 2}+2 / 3 \bar{\zeta}_{1} 1^{3 / 2}\right) B i\left(\zeta_{2}\right)\right\}\right]$ is needed to patch on to the left hand expansion. The result follows from this and patching symmetry.

${ }^{15}$ If one extracts out the $\phi$ dependence one has to face up to an equation in which poles and zeros are closely separated. 
particular integral terms compared with the first order solution if the boundary layer function is given by

$$
\int_{0}^{u}\left(\left|\frac{u-\epsilon S}{u}\right|\right)^{1 / 2} d u=\int_{0}^{x}|g(x, \epsilon)|^{1 / 2} d x
$$

where $S$ is defined by $\epsilon S=u(\omega)$ where $\omega$ is the zero of $g(x, \epsilon)$, and although the $\epsilon^{2}$ term in fact gives rise to particular integrals of order $\epsilon$ such terms are too small to influence the dominating equation. With $S$ and $\zeta$ defined as above the equation for $y$ becomes,

$$
y_{\zeta \zeta}-(1-S / \zeta) y=-\epsilon\left[2 y_{x \zeta} / u_{x}+y_{\zeta} u_{x x} / u_{x}^{2}-\epsilon y_{x x} / u_{x}^{2}\right]
$$

thus $y_{\zeta \zeta}-(1-S / \zeta) y=0$, with $\zeta$ defined by (3.2) is the required related or dominating equation ${ }^{16}$ and one can use any one of a number of techniques to estimate the error term. One could, of course, proceed as in Sec. 2 and obtain an asymptotic expansion; however, in this case the process would be extremely laborious.

\section{REFERENCES}

1. J. J. Mahony, Aust. Math. Soc. 2, 440-463 (1962)

2. R. E. Langer, Trans. Amer. Math. Soc. 33, (1931), 23 ibid 51, (1937) 669 and many others

3. S. Kaplun and P. A. Lagerstrom, J. Math. and Mech. 6, 585 (1957)

4. I. Proudman and J. R. S. Pearson, J. Fluid Mech. 2, 237 (1957)

5. F. W. J. Olver, Phil. Trans. A. 247, 367-369 (1954)

6. N. D. Fowkes, Ph.D. Thesis submitted 1965, Queensland University

7. R. E. Langer, Trans. Amer. Math. Soc. 90, 113-142 (1959)

8. N. D. Kazarinoff, Arch. Rat. Mech. Anal. 2, 129 (1958)

9. F. W. J. Olver, J. Res. Nat. Bur. Standards B63, 131-169 (1959)

10. J. Heading, Phase-integral methods (Methuen Monograph), (1962)

11. M. J. Lighthill, Phil. Mag. 40, 1179 (1949)

12. J. J. Mahony, Resonance in almost linear systems (to be published)

13. J. Cochran, Ph.D. Thesis Stanford University

14. J. D. Cole and J. Kevorkian, Nonlinear differential equations and nonlinear mechanics, Academic Press, N. Y.

15. R. E. Langer, Boletin de la Sociedad Mathematica Mexicana, 1960

${ }^{16}$ The solutions of this equation are confluent hypergeometric functions. 